\title{
Art-Photographic Detail Enhancement
}

\author{
Minjung Son ${ }^{1} \quad$ Yunjin Lee $^{2} \quad$ Henry Kang $^{3} \quad$ Seungyong Lee $^{1}$ \\ ${ }^{1}$ POSTECH $\quad{ }^{2}$ Ajou University $\quad{ }^{3}$ University of Missouri - St. Louis
}

\begin{abstract}
We present a novel method for enhancing details in a digital photograph, inspired by the principle of art photography. In contrast to the previous methods that primarily rely on tone scaling, our technique provides a flexible tone transform model that consists of two operators: shifting and scaling. This model permits shifting of the tonal range in each image region to enable significant detail boosting regardless of the original tone. We optimize these shift and scale factors in our constrained optimization framework to achieve extreme detail enhancement across the image in a piecewise smooth fashion, as in art photography. The experimental results show that the proposed method brings out a significantly large amount of details even from an ordinary low-dynamic range image.
\end{abstract}

Categories and Subject Descriptors (according to ACM CCS): I.3.3 [Computer Graphics]: Picture/Image Generation-Display algorithms

\section{Introduction}

Detail enhancement of digital photographs often provides clarity in conveying shape and structure information. For instance, emphasizing shape and surface detail in medical images may lead to swift and accurate diagnoses. A common approach to detail enhancement involves the decomposition of image into base layer and detail layer, where the base layer accounts for the large-scale features while the detail layer records fine-scale intensity variations. Detail enhancement then reduces to a simple task of boosting (scaling) the detail layer before it is recombined with the base layer. Note however that the amount of scaling to increase details is bounded by the dynamic range of the display device. Consequently, if we apply this tactic to an ordinary low-dynamic range (LDR) image, the resulting image may not capture sufficient details, particularly in very dark (or bright) regions.

In art photography, such exaggerated depiction of finescale detail is often used to elicit aesthetic response from the viewer (see Fig. 1). For example, Dave Hill uses meticulous staging and lighting to synthesize a photograph in which local contrast is significantly exaggerated to a point where the image looks somewhat hyper-realistic, that is, non-photorealistic yet highly detailed [Hil11]. He accomplishes this by taking pictures of individual scene components under different lighting conditions (often to exaggerate details), which are then composited together. Several photographers, including Trey Ratcliff, obtain similar effects by using a high dynamic range (HDR) image, or equivalently, multiple photographs of the same scene taken under different exposures, then selecting the best exposure image for each object/region that can clearly display the structure within it. Therefore, significant details can be revealed even in a supposedly very dark or bright region, creating the aforementioned hyper-realistic effect. The key to generate such synthetic photographs is the ability to handle the lighting conditions of individual regions/objects separately. In other words, the photographer must be given region-specific control to increase the dynamic range of each region as well as the amount of detail therein.

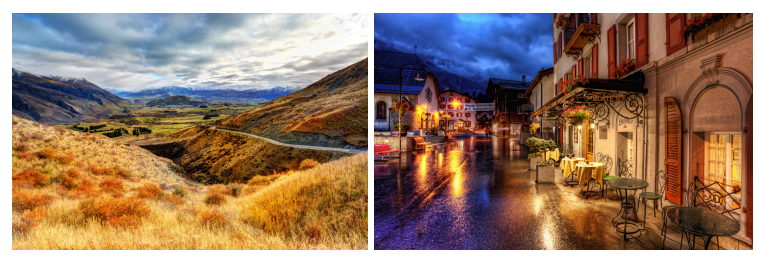

Figure 1: Art photographs by Trey Ratcliff (HDR imaging)

Based on this observation, we propose a novel approach to enhancing details of a single LDR image. The central idea is to introduce a piecewise smooth tone transform model to obtain extremely exaggerated local contrast for each region in the image (à la art photograph) while keeping the resulting tone within the target dynamic range. Our tone transform 
function consists of scaling and shifting operations, a departure from previous detail enhancement approaches that rely on scaling only. The shift operation allows the base layer to be restructured in a piecewise smooth fashion and plays a key role in bringing out details even in some highly dark or bright areas. We use a constrained optimization framework to compute these scale and shift factors that are optimum for each region in boosting details. We also show that our detail enhancement framework lends itself to a simple yet intuitive scheme to control the degree of detail enhancement based on linear interpolation.

The contributions of our paper are summarized as follows.

- A novel approach to single-image detail enhancement: Inspired by art-photographic techniques, we introduce a piecewise smooth tone transform model which emulates art-photographic detail enhancing process and reveals a significantly large amount of details even from a single LDR image.

- Region-specific detail exaggeration: Our tone transform function along with the optimization framework aims to bring out extreme details in each region, as in art photography, via the interplay between detail scaling and base shifting. The resulting image often has the hyper-realistic look of a typical art photograph.

- An interpolation-based level-of-detail control: Our framework enables an intuitive level-of-detail control method based on linear interpolation between the source and the detail-extremized images.

\section{Related Work}

In computational photography, the goal of detail enhancement is to bring out fine-scale shape details by exaggerating local contrast, while reducing large-scale intensity variations to make sure the overall tonal range does not exceed that of the display medium [FAR07]. Manipulating image detail often requires the decomposition of image into base layer and detail layer, followed by the detail coefficients being scaled up and then recombined with the base layer.

Such image decomposition typically involves edgepreserving image smoothing operator, for instance bilateral filter [DD02], which outperforms linear filters in reducing halo artifacts near edges. Bae et al. [BPD06] used this bilateral image decomposition for mimicking the photographic look of a highly detailed image. Fattal et al. [FAR07] generated a detail-exaggerated look of an object via multi-scale bilateral decomposition of a multi-light image collection (MLIC). Farbman et al. [FFLS08] showed that weighted least squares (WLS) operator could outperform bilateral filter in progressive coarsening of images and multiscale detail extraction. Subr et al. [SSD09] defined detail as oscillations between local extrema to better distinguish between high-contrast, fine-scale features from real edges. Paris et al. [PHK11] showed Laplacian pyramid can be used for successful detail enhancement by manipulating gradients at each scale of the pyramid. Gastal and Oliveira [GO11] proposed an efficient domain transform method to perform image decomposition and detail enhancement in real time. Xu et al. [XLXJ11] recently introduced an $L_{0}$ gradient minimization operator that globally optimizes the smoothing result by controlling the number of non-zero gradients in the image. In this paper, we use a modified $L_{0}$ smoothing algorithm to obtain a piecewise smooth image decomposition that facilitates region-based detail enhancement.

The problem definition of detail enhancement (and also its process) bears a close resemblance to that of HDR tone mapping, which generally refers to compressing the dynamic range of an HDR image so it can be displayed on an LDR device with sufficient details. In fact, many existing tone mapping operators, including the edge-preserving smoothing filters discussed in the previous paragraph, can be used on LDR input images for the purpose of bringing out more details [FLW02, LSA05, FFLS08, SSD09, PHK11, XLXJ11] albeit to a much lesser degree than when an HDR input is used. The difficulty of LDR detail enhancement is that the inherently low dynamic range leaves little room for expanding details, especially in very dark or bright regions. We address this limitation by introducing an adaptive and flexible tone transform model which enables a wider range of tone variation for each region in the image.

Some techniques preserve details by managing the exposure of image regions. Mertens et al. [MKR07] suggested blending multiple-exposure images guided by weights computed from saturation and contrast, which resembles the actual process used in art photography. Our method, on the other hand, focuses on bringing out extreme details and does not require multiple input images. Reinhard et al. [RSSF02] applied automatic dodging-and-burning to locally change the exposure and preserve details in dark or light regions, where the selection of proper local scale is imperative in avoiding loss of details or dark rings around strong edges. Kaufman et al. [KLW12] specifically detected faces, skies, and underexposed regions, and corrected their exposures to enhance the details in them.

Our approach is somewhat related to reverse tone mapping operators (rTMOs) that perform LDR to HDR image conversion [BLDC06, $\mathrm{RTS}^{*} 07, \mathrm{MAF}^{*} 09$ ], in that both rTMOs and ours start from LDR input images and look to expand the dynamic range of image regions in the process of enhancing details. However, the basic goal of rTMOs is to estimate an HDR image that best matches the LDR input, and hence focus on minimizing unnatural tone or objectionable artifacts in the result rather than creating hyper-realistic look. In addition, our method does not change the total dynamic range of the input.

Also worth mentioning are techniques that generate artistic or photographic effects from images to reproduce artworks in the real world [DS02, WOG06, EWK $\left.{ }^{*} 13\right]$. Among 


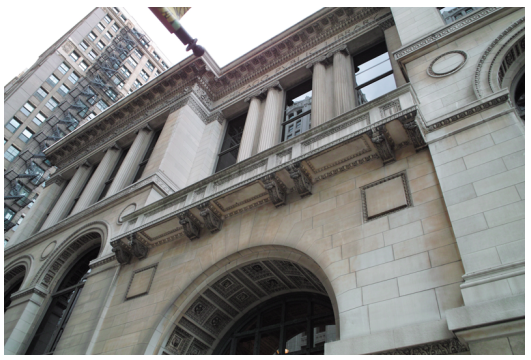

(a) Input

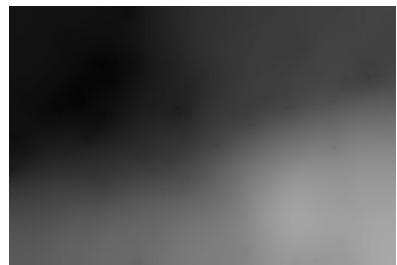

(d) Scales $s$ of (b)

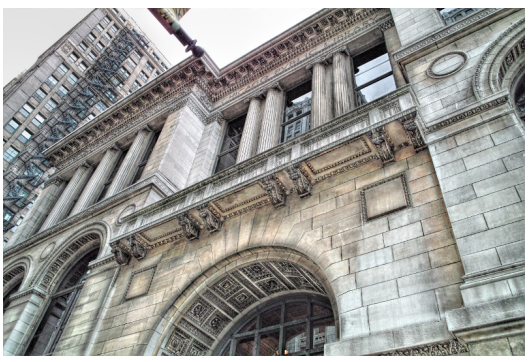

(b) Globally smooth $s$ and $t$

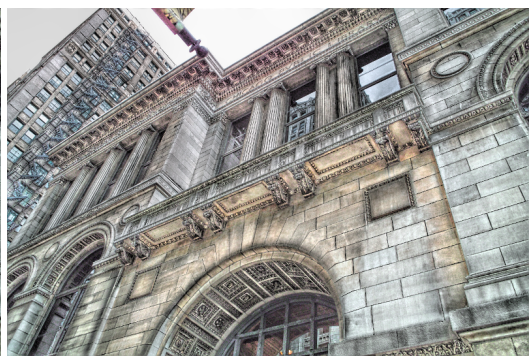

(c) Piecewise smooth $s$ and $t$

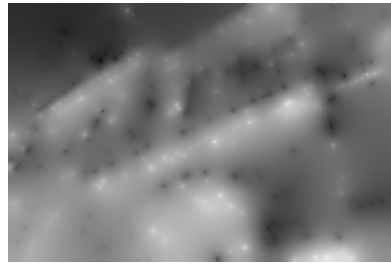

(e) Shifts $t$ of (b), normalized

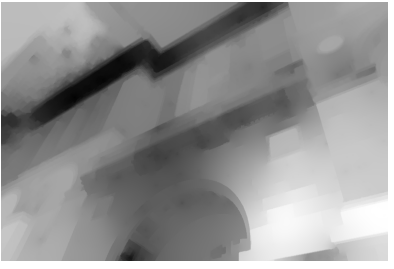

(f) Scales $s$ of (c)

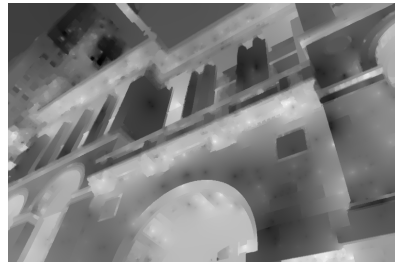

(g) Shifts $t$ of (c), normalized

Figure 2: Changes through smoothness conditions for scaling $s$ and shift $t$ in our tone transform model

them, Echevarria et al. [EWK $\left.{ }^{*} 13\right]$ presented a computational method to simulate analog photographic techniques such as wet plate collodion or cyanotypes, which could exhibit detail-enhanced looks.

\section{Tone Transform Model}

Following the convention in detail enhancement, we decompose the input image $I$ into base layer $B$ and detail layer $D$, where $B$ is a smoothed version of $I$, and $D=I-B$. Let $D_{i}$ denote the detail coefficient at each pixel $i$, that is, $D_{i}=I_{i}-B_{i}$. A simple way of enhancing detail would be to boost the detail coefficients $D_{i}$ as follows:

$$
I_{i}^{\prime}=B_{i}+D_{i}^{\prime}=B_{i}+s_{i} D_{i}
$$

where $s_{i}$ is a scale factor. Note however that the possible range of $s_{i}$ is bounded by the input tone value at $i$ because $I_{i}^{\prime}$ cannot exceed the maximum dynamic range of the display device. This could greatly limit one's ability to enhance detail, especially in the already dark or bright regions. We mitigate this problem by the following modification to the tone transform model:

$$
I_{i}^{\prime}=B_{i}^{\prime}+D_{i}^{\prime}=\left(B_{i}+t_{i}\right)+s_{i} D_{i},
$$

where $t_{i}$ denotes the amount of vertical shift applied to the base layer $B_{i}$. In a nutshell, this model transforms the tone in two ways; shifting the base tone by $t$ and scaling the detail by $s$. In a dark region, a positive $t_{i}$ would brighten the base $B_{i}$ and thus help accommodate a larger $s_{i}$. Similarly, a negative shift would be desirable in a highly bright region.

Eq. (1) embodies the basic principle of art photography, that is, independent control of brightness in each region. The art photographers achieve this by using multiple shots taken with different lights/exposures, so that each object or region could be supplied with a big enough dynamic range to show full details. In this context, scaling would be analogous to adjusting the amount of light/exposure while shifting controls the overall brightness of the scene.

Suppose $t_{i}$ and $s_{i}$ are fixed for all pixels in $I$, and call it a globally constant tone transform. Then most of the original scene structure (which we measure by the location of intensity jumps) would be preserved. However, this globally constant transform has a severely limited range for detail enhancement where $s$ will most likely stay close to one, because an ordinary image often already contains a close-tofull dynamic range.

This brings us to a globally smooth tone transform, where $t_{i}$ and $s_{i}$ may smoothly vary across the image. The smooth variation of $t_{i}$ and $s_{i}$ again ensures preservation of scene structure everywhere, since smooth $t_{i}$ and $s_{i}$ would mean little or no change in the location of intensity jumps in the image. Now $s_{i}$ has a larger room to maneuver and thus brings out more details (Fig. 2(b)), but is still constrained by the requirement to smoothly vary for all pixels.

To relax this constraint, we emulate the region-based brightness control in art photography. That is, we allow piecewise smooth variation of $s_{i}$ and $t_{i}$, and thus different scaling and shift values for each region/object. This means that we allow a discontinuity in $s_{i}$ and/or $t_{i}$ values at object boundaries, which however causes little harm in practice because the human visual system is not sensitive to any dis- 
continuity of information occurring where there is already a large intensity jump. This piecewise treatment of $s_{i}$ and $t_{i}$ allows each homogeneous region to have a larger tonal range, and thus more aggressive detail enhancement (Fig. 2(c)). Sections 4 and 5 discuss how to enable piecewise smooth application of our tone transform model.

\section{Detail and Base Decomposition}

For our tone transform, an ideal base layer $B$ obtained from $I$ would have a constant tone within each homogeneous region while preserving the shape of the edges as closely as possible to those in $I$. Note that there are two important requirements involved. First, a piecewise constant base is a good fit for our purpose because it naturally promotes piecewise constant (or piecewise smooth) detail enhancement by establishing the boundaries (fences) between heterogeneous regions. In this context, the base layer $B$ provides a soft segmentation of the input image $I$. Second, the edges captured in the base layer $B$ must match their original edges in $I$ in terms of shape and location, otherwise could result in artifacts near edges such as halo and gradient reversal, after tone transform for detail enhancement.

In our approach, we perform $L_{0}$ smoothing [XLXJ11] on $I$ to obtain $B$. This operator generates a set of piecewise constant regions and step edges. That is, it already satisfies the first requirement, and thus we modify it to meet the second requirement better. The original $L_{0}$ smoothing is equivalent to solving the following problem:

$$
\min _{B}\left\{\sum_{i}\left(B_{i}-I_{i}\right)^{2}+\lambda \cdot C(B)\right\} .
$$

where $C(B)=\#\left\{i|| \partial_{x} B_{i}|+| \partial_{y} B_{i} \mid \neq 0\right\}$ is a non-zero gradient counting function. This minimization is solved via special alternating optimization with auxiliary variables $h_{i}$ and $v_{i}$, corresponding to $\left|\partial_{x} B_{i}\right|$ and $\left|\partial_{y} B_{i}\right|$ respectively:

$$
\min _{B}\left\{\sum_{i}\left(B_{i}-I_{i}\right)^{2}+\beta\left(\left(\partial_{x} B_{i}-h_{i}\right)^{2}+\left(\partial_{y} B_{i}-v_{i}\right)^{2}\right)\right\}
$$

and

$$
\sum_{i} \min _{h_{i}, v_{i}}\left\{\left(h_{i}-\partial_{x} B_{i}\right)^{2}+\left(v_{i}-\partial_{y} B_{i}\right)^{2}+\frac{\lambda}{\beta} H\left(\left|h_{i}\right|+\left|v_{i}\right|\right)\right\},
$$

where $H\left(\left|h_{i}\right|+\left|v_{i}\right|\right)$ is a binary function having value 1 if $\left|h_{i}\right|+\left|v_{i}\right| \neq 0$ and 0 otherwise. $\lambda$ works as a smoothing parameter that controls the number of non-zero gradients. Therefore a large $\lambda$ increases the number of zero gradients resulting in a piecewise constant $B$ with very few edges. On the other hand, a small $\lambda$ preserves non-zero gradients better.

It is known that overly sharpened or blurred edges in $B$ can cause visual artifacts near edges during detail enhancement (see blue curves in Fig. 3). Since $L_{0}$ operation is designed to generate piecewise constant regions, it may produce many oversharpened edges. Xu et al. [XLXJ11] resolved this via adaptive Gaussian edge smoothing of oversharpened edges. However, some visual artifact may still persist especially along strong edges while amplifying the small differences between $B$ and $I$ in the process of detail enhancement (see red curves in Fig. 3).

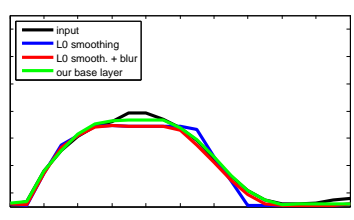

(a) Base layers

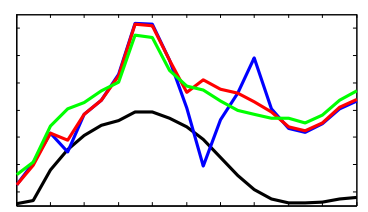

(b) Detail-enhanced signals
Figure 3: Comparison of signals on base layer and their detail-enhanced results using different image decomposition methods

We address this by classifying the sharpened edges into two groups: strong (hard) edges and smooth (soft) edges. Our solution is composed of three passes and is designed to protect both types of edges from oversharpening. In the first pass, we perform the original $L_{0}$ smoothing on $I$ to obtain $B^{1}$ ( $\lambda=0.01$ for all our results). The purpose of this pass is to build an adaptive $\lambda$-map to guide the second pass. The second pass performs $L_{0}$ smoothing on $I$ again but this time using adaptive $\lambda_{i}$ recorded in the $\lambda$-map. Since $\lambda_{i}$ gets progressively smaller near the edges, this avoids oversharpening of strong edges and keeps their original shapes. Let $B^{2}$ denote the outcome of this adaptive $L_{0}$ smoothing. Finally, we adaptively Gaussian-blur the edges in $B^{2}$ as described in [XLXJ11]. The proper Gaussian scale at each pixel is measured by comparing the blurred version of $B^{2}$ and $I$. The outcome of this final smoothing serves as $B$.

The adaptive $\lambda$-map used for the second pass is computed as follows. After the first pass, strong step edges can be easily detected from $B^{1}$ by thresholding $G^{1}$, the gradient of $B^{1}$. We determine edge pixels via thresholding $G_{i}^{1} \geq a$ (default threshold $a=0.2$ for all results), where we set $\lambda_{i}$ as $\varepsilon(\approx 0)$ to properly prserve the original edge shape without oversharpening. We use a small $\varepsilon$ instead of zero to avoid numerical errors. As we move away from edges, i.e., $G_{i}^{1}<a, \lambda_{i}$ should rapidly increase up to $\lambda$ to ensure piecewise flattening of edges within homogeneous region. We model this behavior with the minus half of integral bisquare function [BSMH98],

$$
\rho(u, \sigma)= \begin{cases}\frac{1}{3} & u<-\sigma \\ \frac{u^{2}}{\sigma^{2}}-\frac{u^{4}}{\sigma^{4}}+\frac{u^{6}}{3 \sigma^{6}} & -\sigma \leq u<0 \\ 0 & u \geq 0\end{cases}
$$

The adaptive $\lambda_{i}$ at pixel $i$ is then defined:

$$
\lambda_{i}=3(\lambda-\varepsilon) \rho\left(G_{i}^{1}-a, \sigma\right)+\varepsilon,
$$

with default value of $\sigma=0.1$.

The green curves in Fig. 3 shows that our decompositoin 


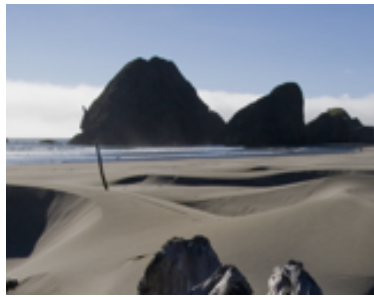

(a) Input

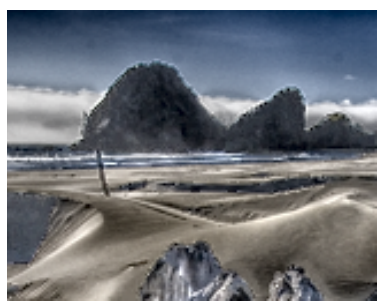

(c) $L_{0}$ smoothing with adaptive blur

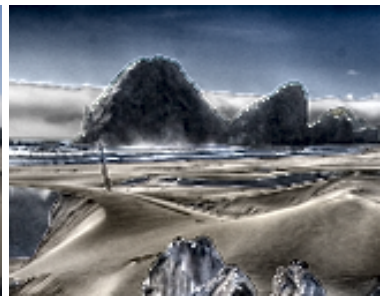

(b) $L_{0}$ smoothing

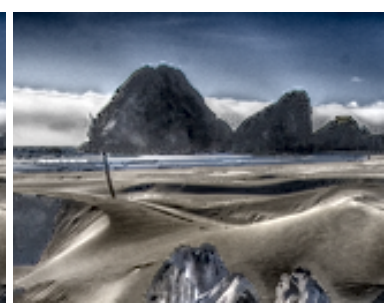

(d) Our method
Figure 4: Comparison of detail enhancements based on different image decomposition methods.

method reduces visual artifacts around strong edges. This is also demonstrated in Fig. 4, where there are sharp edges near mountain silhouettes as well as soft edges near the boundary of sky and clouds. Both types of edges are better preserved with our method.

\section{Detail Maximization}

Once we have the base and detail layers ready, we optimize the tone transform parameters $s_{i}$ and $t_{i}$ to bring out as much detail as possible. We formulate the process of detail enhancement as a constrained optimization problem, which is designed to maximize image detail while preserving scene structure.

\subsection{Detail measure}

Given the decomposition of image $I=B+D$, we measure the amount of detail in $I$ as the squared sum of all detail coefficients $D_{i}$ in the detail layer $D$. Thus for the transformed image $I^{\prime}=s D+B+t$ in Eq. (1), our goal is to maximize $\sum_{i}\left\|s_{i} D_{i}\right\|^{2}$. Note that the base shift $t$ is excluded from this measure because it does not add to the amount of details once the transformation is complete. However, during the optimization process, $t$ does contribute to maximizing $s$ and thus $s D$. To ensure piecewise smooth variation of $s$ and $t$, the detail maximization scheme must simultaneously minimize the smoothness terms $\left\|\nabla s_{i}\right\|^{2}$ and $\left\|\nabla t_{i}\right\|^{2}$. In a perfectly homogeneous region with a flat signal in the base layer, these terms should become zero. i.e., $\left\|\nabla s_{i}\right\|^{2}=\left\|\nabla t_{i}\right\|^{2}=0$, while they are allowed to be flexible at the boundaries between heterogeneous regions. As described in Section 3, this is to encourage region-based control of brightness and detail, inspired by the conventional process of art photography.

The piecewise control is enabled by introducing a weight $w_{i} \in[0,1]$ that is inversely proportional to the gradient magnitude of the base layer $B$. Since $B$ is already piecewise smooth as an outcome of $L_{0}$ smoothing, so should $w_{i}$. We define $w_{i}=K\left(\nabla B_{i}\right)$, where $K(u)$ denotes Tricube function: $K(u)=\left(1-|u / a|^{3}\right)^{3}$ if $|u / a| \leq 1$, otherwise $K(u)=0$. Parameter $a$ determines the value of $u$ where $w_{i}$ drops to zero. We set the default value of $a$ to 0.2 . Within a homogeneous region, the weight is at its $\max w_{i}=K(0)=1$, and it significantly decreases near region boundaries and thus relaxes the smoothness constraint there.

\subsection{Objective function}

Following the description above, we define our objective function to be minimized for maximizing detail as:

$$
\begin{aligned}
f(s, d) & =-\sum_{i}\left\|s_{i} D_{i}\right\|^{2} \\
& +r_{1} \sum_{i} w_{i}\left\|\nabla s_{i}\right\|^{2}+r_{2} \sum_{i} w_{i}\left\|\nabla t_{i}\right\|^{2},
\end{aligned}
$$

with the constraint

$$
0 \leq I_{i}^{\prime}=\left(B_{i}+t_{i}\right)+s_{i} D_{i} \leq 1 .
$$

Note that $w_{i}$ is associated with the smoothness terms for each pixel $i$. We constrain the output image $I_{i}^{\prime}=s_{i} D_{i}+B_{i}+t_{i}$ to have a tonal range between 0 and 1 . This range constraint keeps $s_{i}$ and $t_{i}$ from going infinite and their smoothness terms further limit any drastic change of $s_{i}$ and $t_{i}$ in the local neighborhood except at region boundaries.

Since the function $f(s, d)$ is quadratic and the inequality constraints for the tonal range of the output pixels are linear, this problem can be solved by many standard methods. We used Matlab's quadratic problem solver to obtain $s_{i}$ and $t_{i}$ values that minimize $f(s, d)$. For convex problem condition, we constrain $r \cdot w_{i}$ to be larger than 2 by modifying $w_{i}$ to $K\left(\nabla B_{i}\right)+2 / r$. To achieve piecewise constant scales and shifts, we use large values of $r_{1}$ and $r_{2}$ (with default value $r_{1}=200$ and $\left.r_{2}=500\right)$.

\subsection{Detail control via interpolation}

Once the optimized image $I^{\prime}$ has been obtained, we may optionally use it as an upper bound to control the amount of detail enhancement via linear interpolation with the input image $I$. We find this interpolation-based level-of-detail control scheme to be simple, intuitive, and stable.

Eq. (3) shows the linear interpolation between images $I$ and $I^{\prime}$ using parameter $\mu \in[0,1]$.

$$
\begin{aligned}
I_{\mu} & =\mu I^{\prime}+(1-\mu) I \\
& =\mu(s D+B+t)+(1-\mu)(D+B) \\
& =(\mu s+(1-\mu)) D+B+\mu t
\end{aligned}
$$




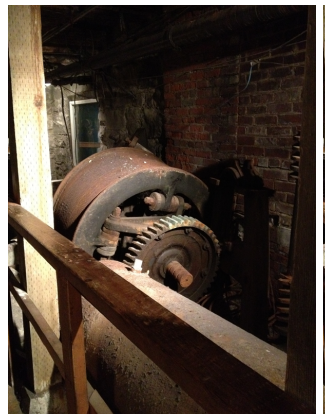

(a) $\mu=0.0$ (input)

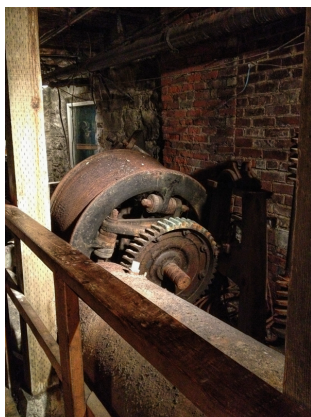

(b) $\mu=0.25$

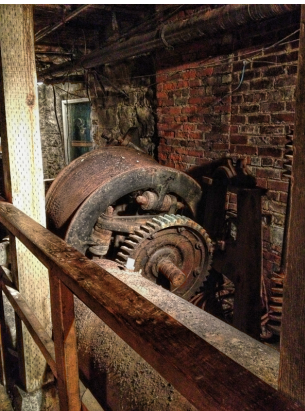

(c) $\mu=0.5$

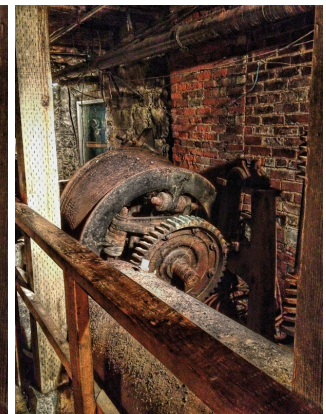

(d) $\mu=0.75$

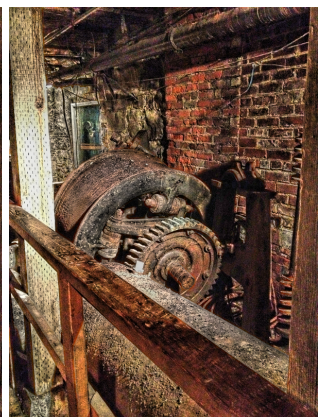

(e) $\mu=1.0$

Figure 5: Detail control with interpolation using parameter $\mu$

Dealing with parameter $\mu$ is more intuitive than, say, having to directly control the scaling factor $s_{i}$ to fine-tune a desired level of detail. Note that it is still a region-adaptive scheme as $I^{\prime}$ has been created in a region-adaptive fashion. Eq. (3) also shows that this interpolation can be interpreted in the context of our tone transform model. That is, the scaling factor for $D$ is linearly interpolated between 1 and $s$, and shift $\mu t$ of $B$ is linearly interpolated between 0 and t. Fig. 5 shows example results generated via our interpolation scheme.

\section{Experimental Results}

We tested our method on an Intel(R) Core(TM) i7 CPU with 16GB memory using Matlab optimization tool, and it takes about 4 minutes to generate detail-enhanced output for an image size of $512 \times 512$. For each example, CIE Lab (Figs. $4,9,10,12(\mathrm{~b})$, and $12(\mathrm{~d})$ ) or HSV color space (remaining examples) is used for tone conversion and color mapping. The default parameter setting used for all examples in the paper is $r_{1}=200, r_{2}=500$, and $\lambda=0.01$.

The results in Fig. 7 are generated by our detail enhancement framework from the low dynamic range (LDR) test images in Fig. 6. Many of these LDR images contain a large portion of dark or bright areas with unclear image details. Fig. 7 demonstrates that our scheme not only sheds lights on these hidden details but also generates the hyper-realistic and dramatic look commonly seen in art photographs.

Aside from art-photographic image generation, our method is applicable to other types of image enhancement, such as haze removal and medical imaging. The proposed detail enhancement algorithm is particularly useful for medical image enhancement (see Fig. 8) where the hidden details become clear and easily noticeable. Note that the quality of detail enhancement via commercial photo editing software or local histogram equalization [PAA*87] is not nearly as good. While our method is not specifically designed to remove image haze, we find its performance in the presence of haze surprisingly robust and effective (see Fig. 9). This
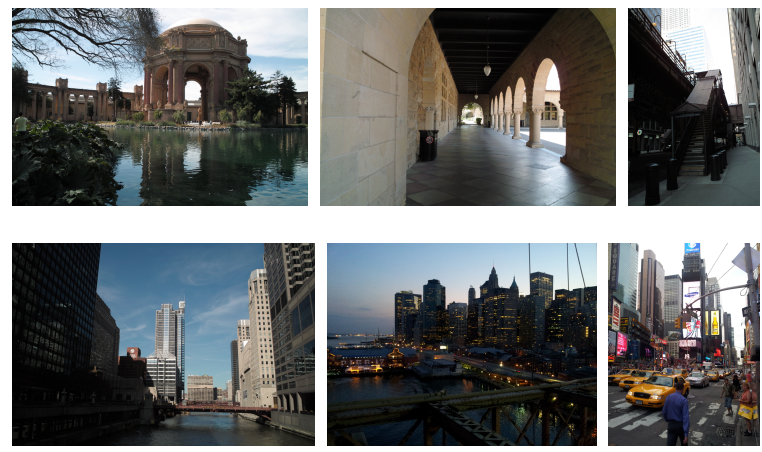

Figure 6: Input LDR images

is attributed to our base shift operator that has the effect of reducing haze, in combination with the extreme expansion of detail coefficients. The default parameter setting of our method worked consistently well for these applications.

Fig. 10 compares previous detail enhancement methods with ours. Again, the input images Fig. 10(a) and (c) are of low-dynamic range. Fig. 10(b) and (d) have been obtained by simply scaling up the detail coefficients by five times and ten times, respectively. Then the left half of the result was remapped to $[0,1]$ afterwards to avoid clipping in dark and bright regions, and thus shows reduced effect of detail enhancement. In Fig. 10(e)-(i) and (k), we applied both tone mapping (range compression) and detail enhancement on the LDR input images. As expected, they all bring out more details but to a lesser degree than from an HDR input, even with considerable effort on parameter tuning. These results were produced using the code available on the authors' webpages with the parameter settings described in Fig. 10. On the other hand, Fig. 10(j) and (1) show that our technique revives a significant amount of details in almost everywhere in the scene, regardless of the original local tone, on account of 


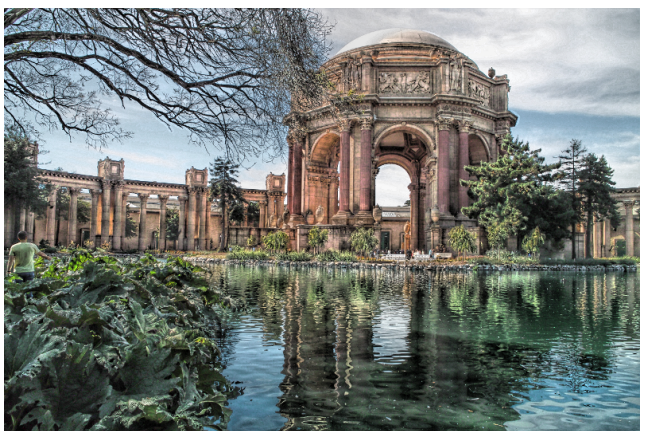

(a)

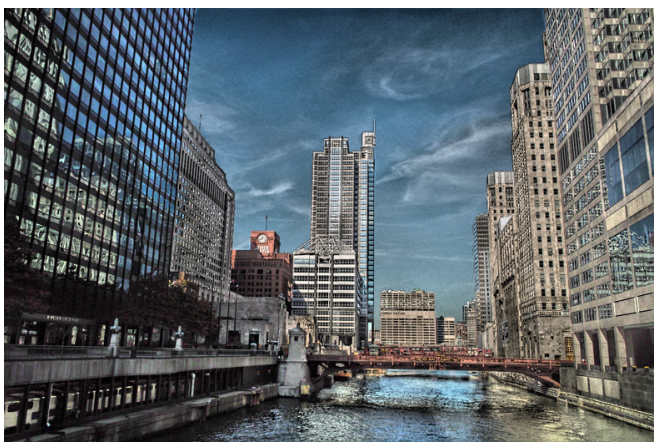

(d)

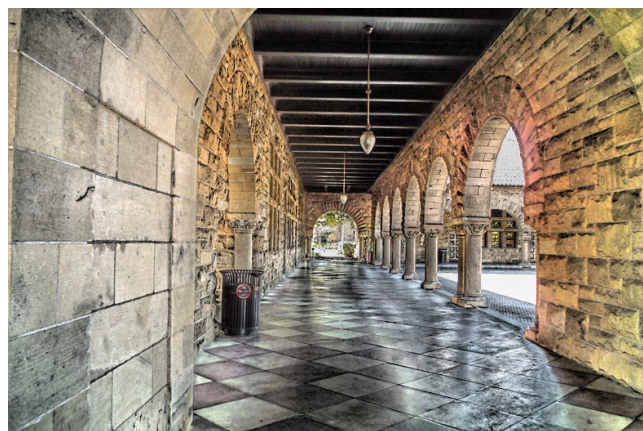

(b)

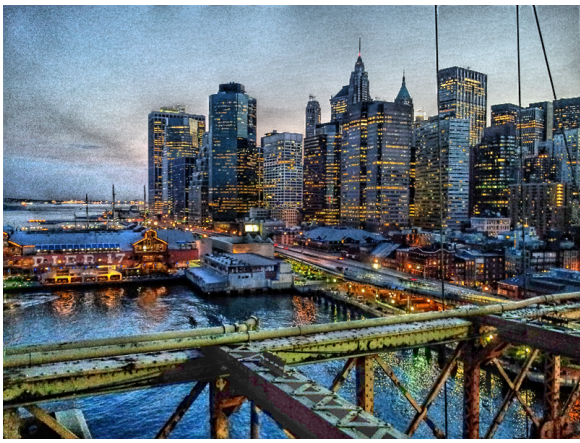

(e)

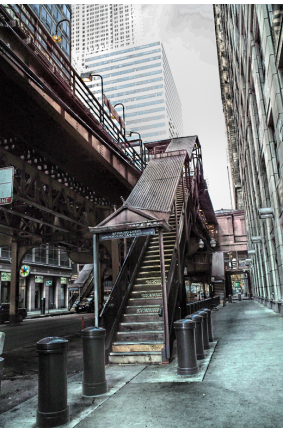

(c)

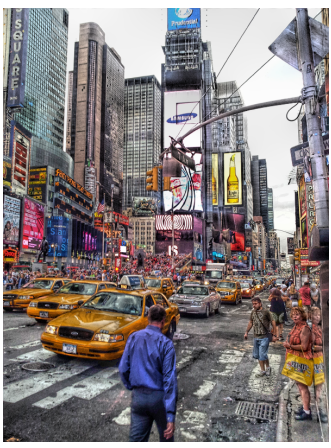

(f)

Figure 7: Output images with extreme detail enhancement

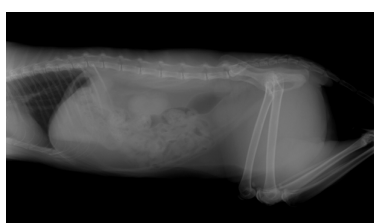

(a) Input

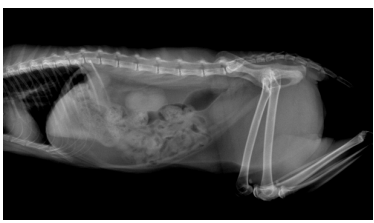

(c) Photoshopped (sharpen filter)

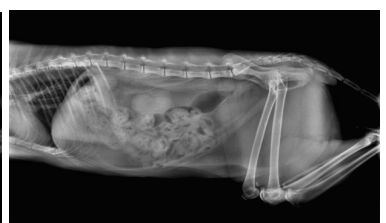

(b) Local histogram equalization

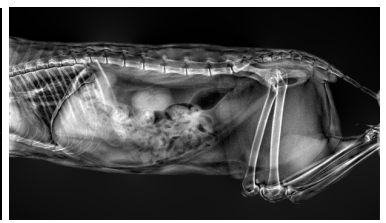

(d) Our method

Figure 8: Medical imaging example

the piecewise shifting of base tone combined with extreme detail boosting.

Fig. 11(b) shows a professional art photograph created by Trey Ratcliff. This image has been synthesized from multiple exposure images of the scene, that is, from an HDR image. On the other hand, our result Fig. 11(d) is obtained from

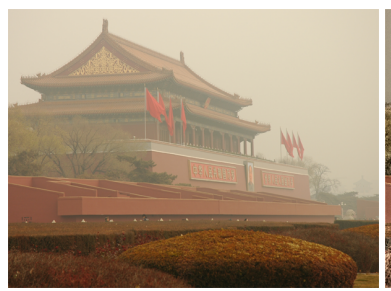

(a) Input

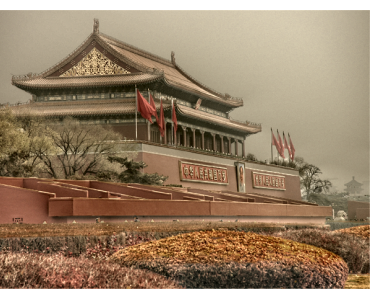

(b) Our method
Figure 9: Haze removal with extreme detail enhancement

Fig. 11(a), an LDR input image of the same scene. Note that our method has managed to bring out comparable or more detail than the professional art photograph generated from an HDR image. In comparison, Fig. 11(c) shows that the stateof-the-art detail enhancement technique [PHK11] does not recover as much detail from the same LDR input image of Fig. 11(a).

\section{Discussion and Future Work}

What sets apart our approach from previous ones lies in the piecewise smooth handling of detail boosting as well as base 


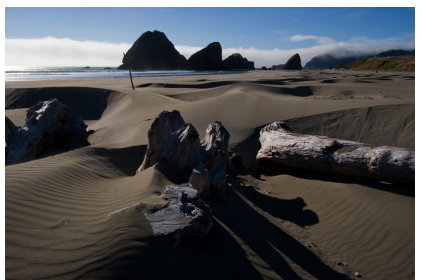

(a) LDR input

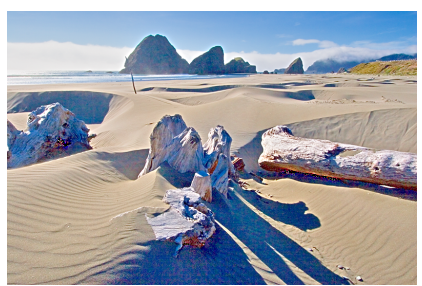

(e) Li et al. [LSA05]

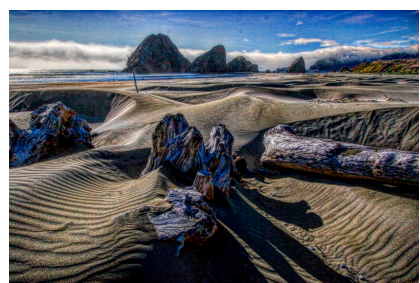

(i) Paris et al. [PHK11]

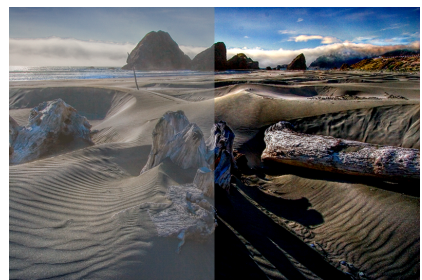

(b) Xu et al. [XLXJ11]

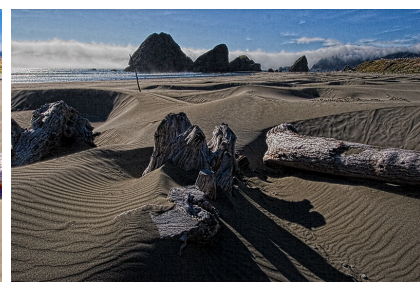

(f) Farbman et al. [FFLS08]

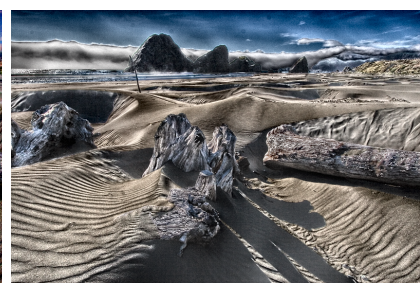

(j) Our method

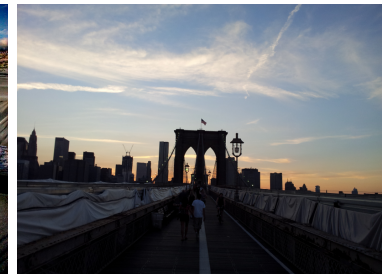

(c) LDR input

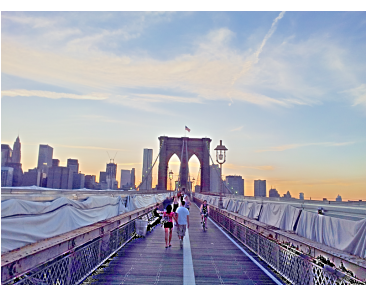

(g) Li et al. [LSA05]

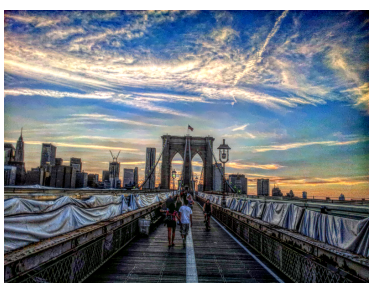

(k) Paris et al. [PHK11]

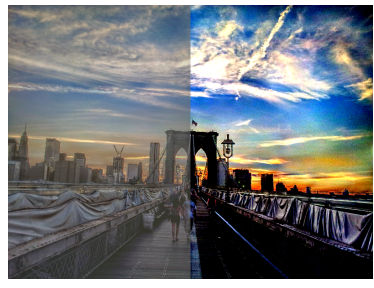

(d) Xu et al. [XLXJ11]

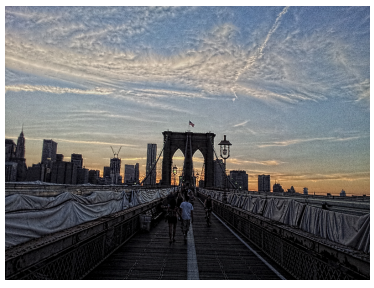

(h) Farbman et al. [FFLS08]

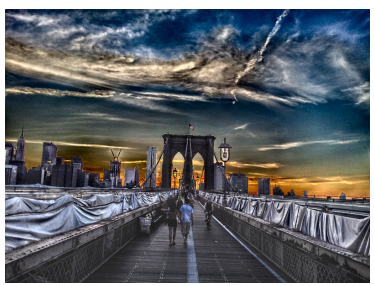

(1) Our method

Figure 10: Comparison with previous methods: $(b),(d)$ scaling up the detail coefficients by five and ten times, respectively, where the left half of the result was remapped to [0,1] afterwards, $(e),(g) \beta=0.6, \alpha=0.7$, endrate $=0.6$, and an Haar wavelet decomposition, $(f),(h) \lambda=\left[\begin{array}{lll}0.05 & 2 & 16\end{array}\right], w=\left[\begin{array}{llll}10 & 10 & 0.75 & 0.8\end{array}\right],(i),(k) \alpha=0.15, \beta=0, \sigma_{r}=0.4$.

shifting. It all begins with the piecewise smooth decomposition of the image $I$. Therefore, if the decomposition operator (modified $L_{0}$ in our case) generates incorrect soft segmentation in the base layer $B$, the quality of detail enhancement would be degraded. For example, Fig. 12(b) shows a case where the sky is segmented into multiple regions and toneadjusted by different amounts. This artifact may be attenuated by our linear interpolation scheme but at the cost of losing some detail elsewhere.

While our flexible and aggressive region-based tone shifting is the key to bringing out extreme details throughout the image, it may also introduce a side effect of brightness reversal between neighboring regions. Fig. 12(d) shows an example where the shadow turns brighter than the unshadowed neighborhood, and the body of the lighthouse turns darker than the sky. We observe that this phenomenon usually occurs across object boundaries and therefore rarely causes noticeable visual artifact. Also worth noting is that image noise is often indistinguishable from small-scale detail and thus gets amplified together. Fig. 12(d) reveals some noise and compression artifacts that were hidden in the input image.
The underlying principle of our method, just like any tone mapping or detail enhancement algorithms, is to shrink large contrast in the image while expanding small-scale discontinuities. The difference is that we allow this trade-off to be controlled in a much more flexible and piecewiseindependent way so that the detail enhancement can go truly extreme if desired. On certain situations, however, this may be viewed as a limitation when the algorithm ends up eliminating mid-level contrast in an effort to bring out too much detail from dark regions (e.g., loss of contrast between sand and rocks in Fig. 10(j)). Again, our interpolation scheme may be used to help alleviate this effect and also control the trade-off.

The task of detail boosting is inherently prone to generate certain artifacts around strong edges such as halo or gradient reversal. Due to the imperfect nature of image decomposition, it is impossible to entirely eliminate such artifacts from all images, especially when the detail boosting goes extreme as in our case. While Section 4 described how our image decomposition strategy helps reduce those artifacts, some of them can still persist especially around edges separating two 


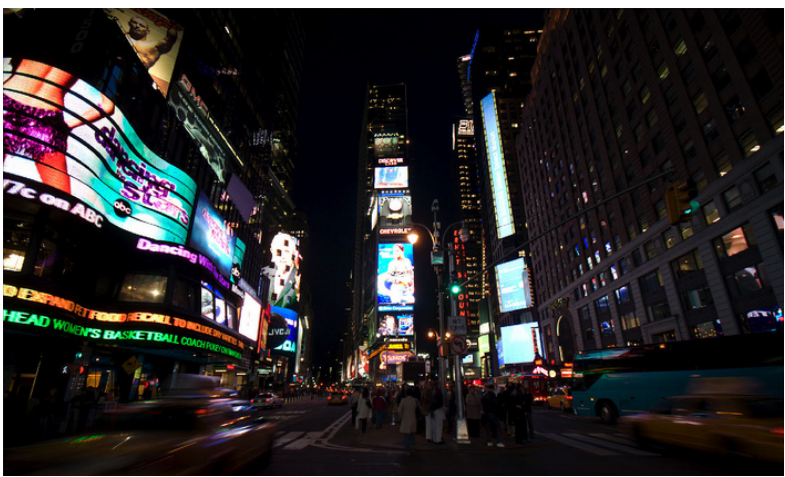

(a) LDR input

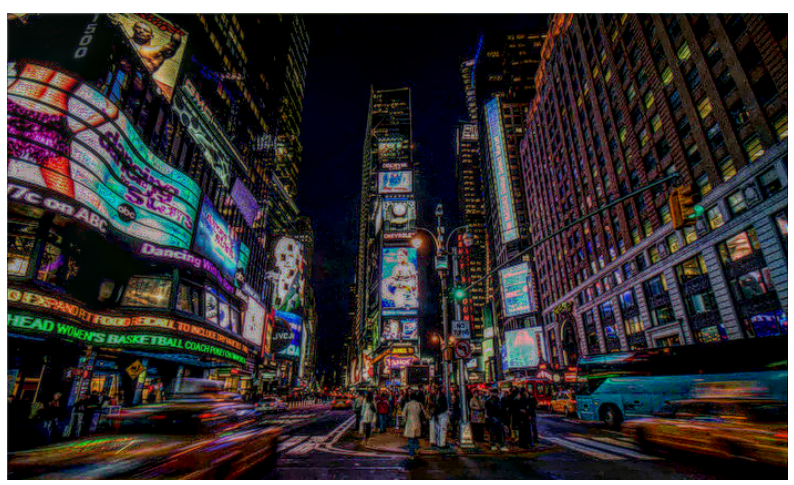

(c) Paris et al. [PHK11], $\alpha=0.15, \beta=0, \sigma_{r}=0.4$

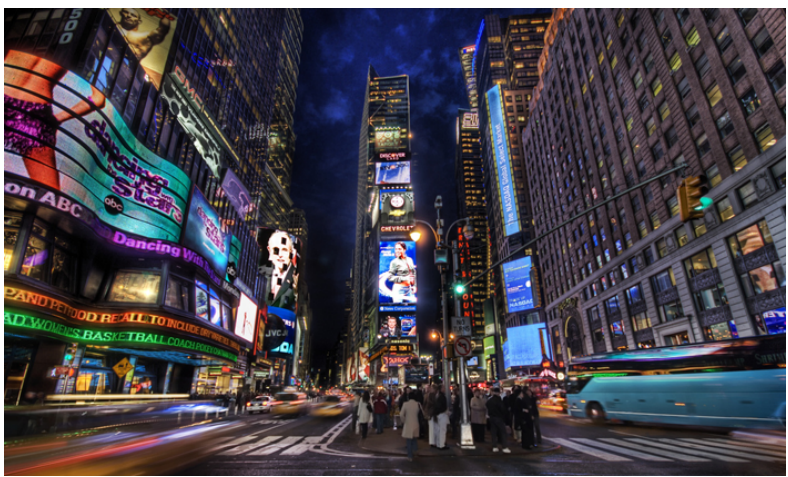

(b) HDR imaging by Trey Ratcliff using multiple exposure images

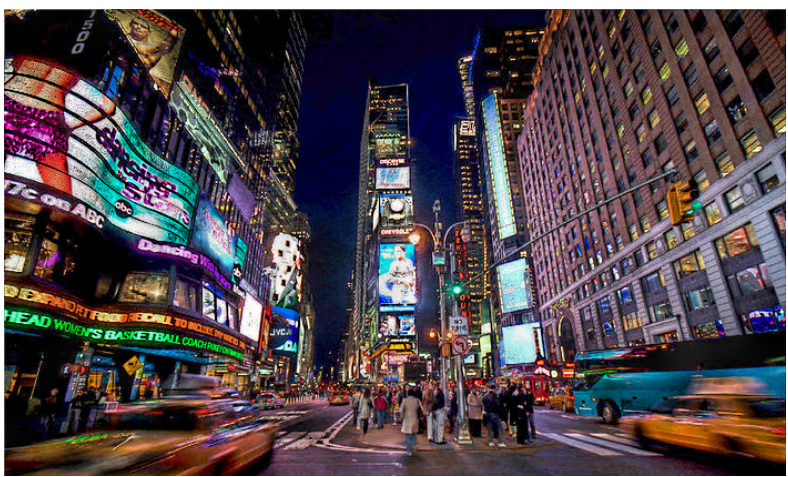

(d) Our method

Figure 11: Comparison with art photograph

severely dissimilar regions in terms of colors and intensities, as shown in Fig. 10(j) and (1). In many of the test images we used (as in Fig. 7), however, the visual quality degradation from such artifacts was insignificant.

Our method may be applicable to HDR tone mapping as well. Fig. 13 shows an HDR tone mapping result based on our detail enhancement scheme. In contrast to the previous approach in which the base layer is first range-compressed and then combined with processed details, we tried to emulate tone mapping by scaling the details and shifting the base layer within the target dynamic range. However, as shifting alone did not fully account for the range compression of the base layer, the details would get insufficiently amplified in some regions. For future work, we plan to find an effective way to control scales and shifts for HDR images.

Most of the computation in our detail enhancement scheme takes place in the luminance channel. A possible future extension would be to take full color components into account in defining and processing details, so the image detail would be maximized not only in terms of the contrast of brightness but also of color, and thus adding vividness to the output. In our current implementation, most of the process- ing time is devoted to the optimization phase. A use of specialized optimization code along with a careful implementation would lead to significant speed-up. We also plan to develop a multi-scale approach to our optimization framework for further acceleration. In this case, the base shift and detail boosting at the coarse level would have to be propagated to the progressively finer levels. An ideal implementation of art photography would require semantically correct image decomposition followed by separate manipulation of the segmented scene components, which may benefit from employing a semantic image segmentation algorithm $\left[\mathrm{AHG}^{*} 12\right]$.

\section{Acknowledgements}

We thank Trey Ratcliff (stuckincustoms.com) for permission to use Figs. 1, 11(a), and 11(b) and Flickr users for sharing their images under a Creative Commons license. This research was supported by Basic Science Research Program of NRF (2012R1A1A2042837, 2013R1A1A2011692).

\section{References}

[AHG*12] Arbelaez P., Hariharan B., Gu C., Gupta S., BOURDEV L. D., MALIK J.: Semantic segmentation using re- 


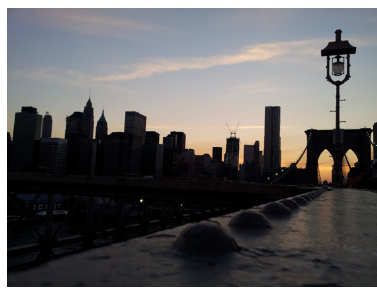

(a) Input

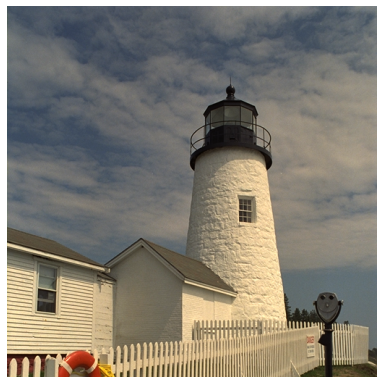

(c) Input

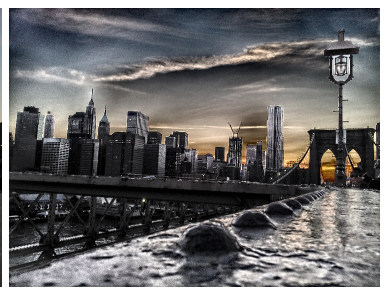

(b) Result

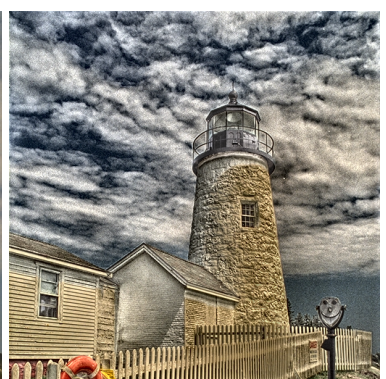

(d) Result
Figure 12: Limitations: (b) sky segmented into heterogeneous regions, $(d)$ brightness reversal and enhanced noise

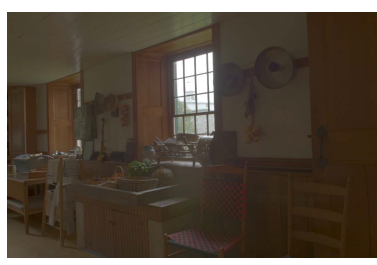

(a) Input

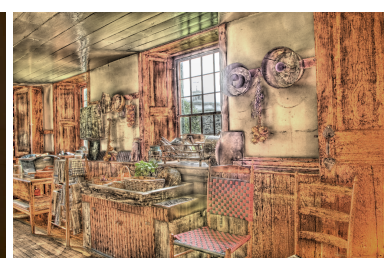

(b) Our result
Figure 13: HDR tone mapping with detail enhancement

gions and parts. Proc. IEEE Computer Vision and Pattern Recognition (2012), 3378-3385. 9

[BLDC06] BANTERle F., LedDA P., Debattista K., Chalmers A.: Inverse tone mapping. In Proc. GRAPHITE 'O6 (2006), pp. 349-356. 2

[BPD06] BaE S., PARIS S., Durand F.: Two-scale tone management for photographic look. ACM Computer Graphics (Proc. SIGGRAPH 2006) 25, 3 (2006), 637-645. 2

[BSMH98] Black M. J., Sapiro G., Marimont D. H., HEEGER D.: Robust anisotropic diffusion. IEEE Trans. on Image Processing 7, 3 (1998), 421-432. 4

[DD02] DURAND F., DORSEY J.: Fast bilateral filtering for the display of high-dynamic-range images. ACM Computer Graphics (Proc. SIGGRAPH 2002) 21, 3 (2002), 257-266. 2

[DS02] DeCARlo D., SANTElla A.: Stylization and abstraction of photographs. ACM Computer Graphics (Proc. SIGGRAPH 2002) (2002), 769-776. 2

[EWK*13] ECHEVARRIA J. I., Wilensky G., KRISHNASWAMY A., Kim B., GUtierreZ D.: Computational simulation of alternative photographic processes. Computer Graphics Forum (Proc. EGSR 2013) 32, 4 (2013). 2, 3

[FAR07] Fattal R., Agrawala M., Rusinkiewicz S.: Multiscale shape and detail enhancement from multi-light image collections. ACM Transactions on Graphics (Proc. SIGGRAPH 2007) 26, 3 (2007). 2

[FFLS08] Farbman Z., FatTal R., Lischinski D., SZEliski R.: Edge-preserving decompositions for multi-scale tone and detail manipulation. ACM Transactions on Graphics (Proc. SIGGRAPH 2008) 27, 3 (2008). 2, 8

[FLW02] FATtAL R., Lischinski D., WERMAN M.: Gradient domain high dynamic range compression. ACM Computer Graphics (Proc. SIGGRAPH 2002) (2002), 249-256. 2

[GO11] Gastal E. S. L., Oliveira M. M.: Domain transform for edge-aware image and video processing. ACM Transactions on Graphics (Proc. SIGGRAPH 2011) 30, 4 (2011), 69:1-69:12. 2

[Hil11] HILL D.: http://vimeo.com/26297793, 2011. 1

[KLW12] KaUfman L., Lischinski D., Werman M. Content-awaare automatic photo enhancement. Computer Graphics Forum 31, 8 (2012), 2528-2540. 2

[LSA05] Li Y., Sharan L., Adelson E. H.: Compressing and companding high dynamic range images with subband architectures. ACM Computer Graphics (Proc. SIGGRAPH 2005) 24, 3 (2005), 836-844. 2, 8

[MAF*09] Masia B., Agustin S., Fleming R. W., Sorkine O., Gutierrez D.: Evaluation of reverse tone mapping through varying exposure conditions. ACM Transactions on Graphics (Proc. SIGGRAPH Asia 2009) 28, 5 (2009), 160:1-160:8. 2

[MKR07] Mertens T., Kautz J., ReEth F. V.: Exposure fusion. Proc. Pacific Graphics 2007 (2007), 382-390. 2

[PAA*87] Pizer S. M., Amburn E. P., Austin J. D., Cromartie R., Geselowitz A., Greer T., Romeny B. T. H., ZIMMERMAN J. B.: Adaptive histogram equalization and its variations. Computer Vision, Graphics, and Image Processing 39, 3 (1987), 355-368. 6

[PHK11] PARIS S., HASINOFF S. W., KaUtz J.: Local Laplacian filters: Edge-aware image processing with a Laplacian pyramid. ACM Transactions on Graphics (Proc. SIGGRAPH 2011) 30, 4 (2011). 2, 7, 8, 9

[RSSF02] Reinhard E., Stark M., Shirley P., Ferwerda J.: Photographic tone reproduction for digital images. ACM Computer Graphics (Proc. SIGGRAPH 2002) 21, 3 (2002), 267276. 2

[RTS*07] Rempel A. G., Trentacoste M., Seetzen H., Young H. D., Heidrich W., Whitehead L., Ward G.: Ldr2hdr: On-the-fly reverse tone mapping of legacy video and photographs. ACM Transactions on Graphics (Proc. SIGGRAPH 2007) 26,3 (2007). 2

[SSD09] Subr K., Soler C., Durand F.: Edge-preserving multiscale image decomposition based on local extrema. ACM Transactions on Graphics (Proc. SIGGRAPH Asia 2009) 28, 5 (2009). 2

[WOG06] Winnemöller H., Olsen S. C., Gooch B.: Realtime video abstraction. ACM Computer Graphics (Proc. SIGGRAPH 2006) (2006), 1221-1226. 2

[XLXJ11] XU L., LU C., XU Y., JIA J.: Image smoothing via L0 gradient minimization. ACM Transactions on Graphics (Proc. SIGGRAPH Asia 2011) 30, 5 (2011). 2, 4, 8 